\title{
Angiogenesis and vasculogenesis as therapeutic strategies for postnatal neovascularization
}

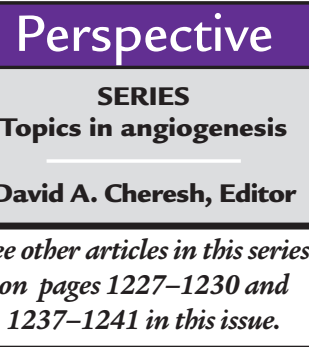

\author{
Jeffrey M. Isner and Takayuki Asahara \\ Tufts University School of Medicine, and the Divisions of Vascular Medicine \\ and Biomedical Research, St. Elizabeth's Medical Center, Boston, Massachusetts 02135, USA
}

Address correspondence to: Jeffrey M. Isner, St. Elizabeth's Medical Center, 736 Cambridge Street, Boston, Massachusetts 02135, USA. Phone: (617) 789-2392; Fax: (617) 779-6362. E-mail: jisner@opal.tufts.edu.

The therapeutic implications of angiogenic growth factors were identified by the pioneering work of Folkman 2 decades ago (1). His laboratory's work documented the extent to which tumor development was dependent upon neovascularization and suggested that this relationship might involve angiogenic growth factors that were specific for neoplasms. Subsequent investigations have established the feasibility of using recombinant formulations of such angiogenic growth factors to expedite and/or augment collateral artery development in animal models of myocardial and hindlimb ischemia. This novel strategy for the treatment of vascular insufficiency was termed therapeutic angiogenesis (2). More recent data suggests that the basis for native and therapeutic neovascularization is not restricted to angiogenesis but includes postnatal vasculogenesis as well. Data supporting these notions, as well as derivative concepts and concerns, are the subject of this Perspective.

Therapeutic angiogenesis. Preclinical studies established that angiogenic growth factors could promote collateral artery development in animal models of peripheral and myocardial ischemia (reviewed in ref. 3). Morphometric analyses documented that such enhanced vascularity encompassed a range of vessel caliber, from mediumsized arteries viewed by premortem angiography, to increased capillary density demonstrated in postmortem histology. The median range of new vessel growth, however, appears skewed toward smaller-caliber arteries less than $180 \mu \mathrm{m}$ in diameter. Indeed, there is good reason to suspect that a proportion of newly recognized mediumsized arteries may develop as a result of arteriogenesis, i.e., in situ proliferation of preexisting arteriolar connections into larger collateral vessels (4). Whether such remodeling occurs as a direct result of growth factor modulation or as a flow-mediated response to augmented downstream capacitance remains to be determined.

The angiogenic growth factors employed in these preclinical studies have been administered as recombinant protein or by gene transfer, and include vascular endothelial growth factor (VEGF), fibroblast growth factor-1 (FGF-1), FGF-2, and hepatocyte growth factor (HGF). More recently, the angiotensin-converting enzyme (ACE) inhibitor quinaprilat and naked DNA encoding for the transcription factor hypoxia inducible factor-1 (HIF-1) have each been shown to enhance neovascularization in the rabbit ischemic hindlimb model.

The optimal preparation and delivery strategy for therapeutic neovascularization is the subject of ongoing clinical investigation. The potential requirement to maintain a suitably high and local concentration over a period of days to weeks constitutes an advantage for gene transfer versus recombinant protein therapy. While viral vectors (5) may enhance transfection efficiency, and thus yield higher levels of gene expression, this may be less relevant to strategies in which gene products such as VEGF include a signal sequence that permits active secretion from intact cells; previous studies from our laboratory (6) have documented that naked DNA that encodes for a secreted protein - as opposed to proteins that remain intracellular - can yield meaningful biologic outcomes due to paracrine effects of the secreted gene product.

The angiogenic growth factors studied in these preclinical experiments have important features in common. All are mitogens for endothelial cells (ECs), although the proliferative effect of these agents measured in vitro is typically limited to between a 2 - and 4 -fold increase. All have likewise been demonstrated to promote EC migration. Most angiogenic cytokines have been shown to include an autocrine loop for at least one cell type in which their synthesis occurs. When studied for effects on EC survival, nearly all have been shown to inhibit EC apoptosis. VEGF and the FGFs have been shown to upregulate nitric oxide, and in the case of VEGF, experiments performed in mice lacking EC nitric oxide synthase suggested that nitric oxide may act as an important downstream mediator for VEGF (7). Upregulation of matrix proteins, matrix proteinases, and certain adhesion molecules represent additional characteristic features of these growth factors. Furthermore, all have been shown to upregulate a variety of other cytokines, in particular VEGF. This evolving profile implies certain aspects of the cascade of events required for neovascularization.

EC specificity has been considered an important advantage of VEGF for therapeutic neovascularization, because ECs represent the critical cellular element responsible for new vessel formation (1). Recent findings, however, have indicated that non-EC populations (8) may express one or more VEGF receptors. Whether such pleiotropism is required for recruitment of other cellular elements, specifically smooth muscle cells and/or pericytes, or occurs as the consequence of secondary cytokine synthesis by activated ECs is currently unclear. Gene targeting experiments performed in mice suggest that full elaboration and stability of native neovascularization is dependent upon such secondary cytokine expression, including platelet-derived growth factor B (PDGFB) and angiopoietin-1. The extent to which these observations imply that multiple reagents must be concurrently administered for therapeutic strategies to recapitulate and/or optimize native angiogenesis remains to be clarified. 

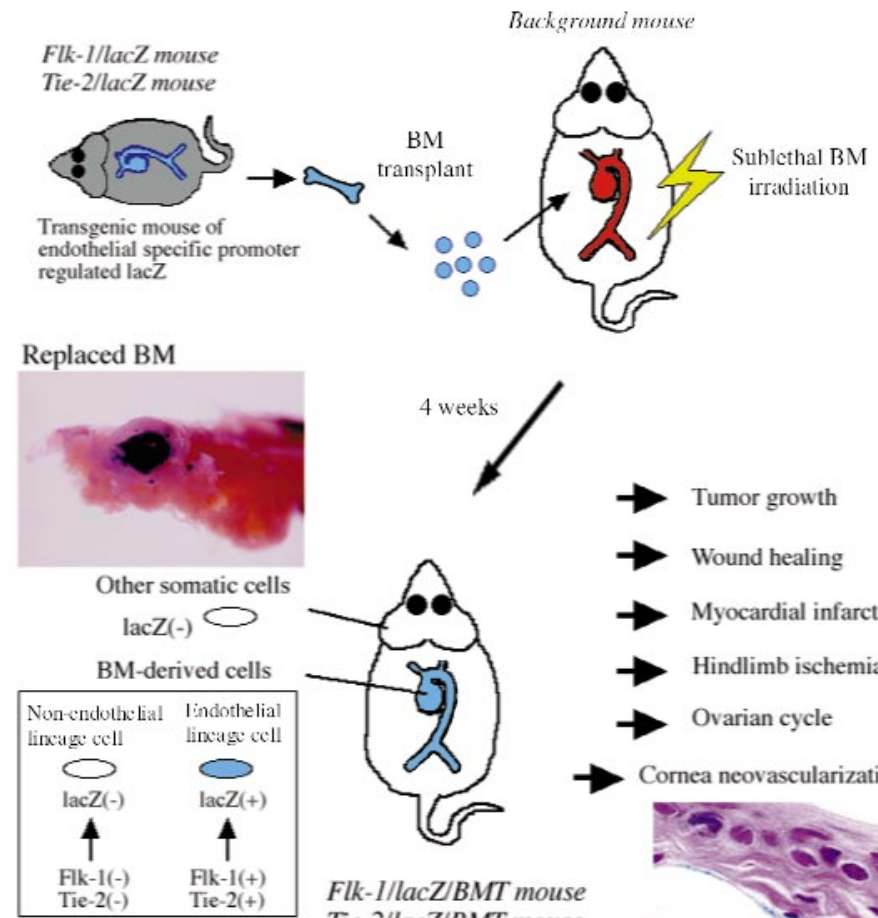

Tie-2/lacZ/BMT mouse

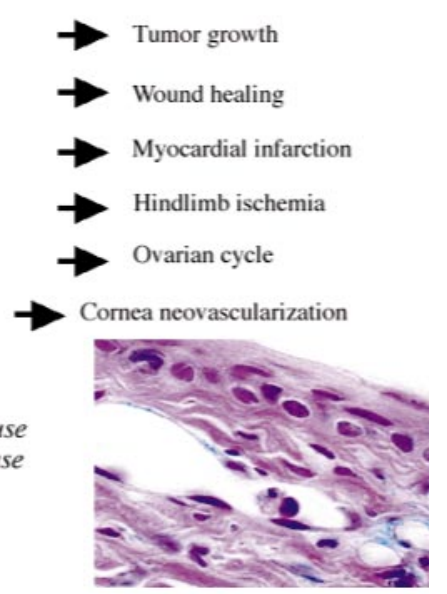

Figure 1

BMT model employed to study contribution of postnatal vasculogenesis to neovascularization of ischemic tissues. Transgenic mouse constitutively expressing LacZ gene transcriptionally regulated by an EC-specific promoter, Flk-1 or Tie-2, is used as BM donor. $\mathrm{BM}$ is harvested and transplanted to mouse of same genetic background, in which BM has been sublethally irradiated. After a period of 4 weeks to allow for reconstitution of transplanted BM, recipient mouse undergoes one or more interventions, all of which are intended to serve as stimulus for neovascularization. At arbitrarily selected time points following these interventions, animals are sacrificed and the respective tissues stained with $X$-gal to histologically identify cells in which expression of $\beta$-galactosidase produces blue cells. Use of an EC-specific promoter permits identification of blue cells, which have incorporated into foci of neovascularization as endothelial lineage cells.

Proof that therapeutic angiogenesis could be successfully extended to human subjects was first demonstrated using gene transfer of naked DNA encoding for VEGF (phVEGF) for the treatment of critical limb ischemia (9). Using a dose-escalating design, treatment was initiated with $100 \mu \mathrm{g}$ of phVEGF. Three patients presenting with rest pain (but no gangrene) and treated with $1,000 \mu \mathrm{g}$ of phVEGF were subsequently shown at 1-year follow-up to have improved blood flow to the ischemic limb as well as no rest pain. With the increase in dose of phVEGF to $2,000 \mu \mathrm{g}$, angiographic and histologic evidence of new blood vessel formation became apparent (9). More recently, the use of intramuscular gene transfer, employed initially as a means of treating patients in whom vascular disease in the ischemic limb was too extensive to permit an intra-arterial approach, achieved marked improvement in collateral vessel development in patients with critical limb ischemia (10). Objective findings of bioactivity in this preliminary report included improvement in the ankle-brachial index, angiographic evidence of newly visible collateral blood vessels, and demonstration by magnetic reso- nance angiography of improved lower extremity blood flow. Ischemic ulcers healed or markedly improved in 4 of 7 limbs, including successful limb salvage in 3 patients recommended for below-knee amputation.

Successful application of both gene transfer and recombinant protein administration for the treatment of myocardial ischemia in human subjects was reported in 1998. Gene transfer involved direct intramyocardial injection of phVEGF as sole therapy for myocardial ischemia refractory to conventional therapy (11). Among 28 consecutive patients treated with this strategy to date, anginal episodes requiring sublingual nitroglycerin were reduced from nearly 60 per week to less than 3 per week. Objective evidence of improved perfusion was documented by a near doubling of treadmill exercise time and improved myocardial blood flow on stress and resting nuclear perfusion scans. The improvement in perfusion observed at rest is consistent with resolution of hibernating myocardium, a finding that has been recently confirmed using catheter-based electromechanical mapping (Vale, P., and Losordo, D., unpublished data). Recombinant protein administration using FGF-1 has also been reported to augment myocardial revascularization and improve functional status in patients undergoing concurrent coronary artery bypass surgery (12).

In vitro studies have suggested certain mechanisms that may have contributed to the apparent benefit and safety of phVEGF gene transfer in these early trials. While ECs were previously viewed solely as the target for VEGF, it is now clear that ECs subjected to hypoxia can synthesize VEGF as well (13). This autocrine feature of VEGF creates the opportunity for amplifying the effects of even a small amount of exogenous VEGF, as EC proliferation in the ischemic territory creates additional potential cellular sources of VEGF synthesis and secretion. Moreover, the recent observation that VEGF may upregulate its own receptor (VEGFR-2, or KDR) (14) establishes a second basis for autocrine and paracrine amplification.

VEGF has also been shown to inhibit EC apoptosis by activating the serine-threonine protein kinase Akt through a process requiring integrin ligation (15). This finding suggests a mechanism other than mitogenesis by which a net increase in EC viability may be accomplished. Given the limited 2- to 4-fold increase shown for VEGF on cellular proliferation, it is possible that the contribution of enhanced EC survival under conditions of severe ischemia is critical to the proangiogenic effects of VEGF and other angiogenic growth factors.

The preclinical and clinical studies of therapeutic angiogenesis performed to date have repeatedly shown that VEGF-induced angiogenesis is not indiscriminate or widespread but is instead restricted to sites of 


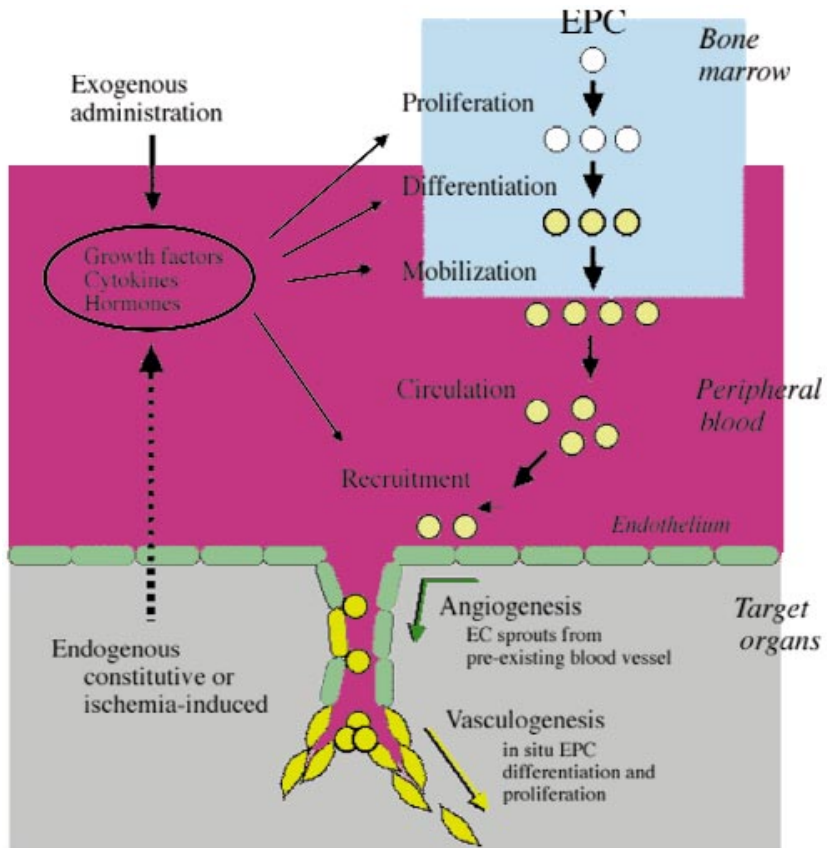

Figure 2

Neovascularization encompasses both angiogenesis and vasculogenesis. Angiogenesis represents the classic paradigm for new vessel growth, as mature, differentiated ECs break free from their basement membrane and migrate as well as proliferate to form sprouts from parental vessels. Vasculogenesis involves participation of BM-derived EPCs, which circulate to sites of neovascularization where they differentiate in situ into mature ECs. Growth factors, cytokines, or hormones released endogenously in response to tissue ischemia, or administered exogenously for therapeutic neovascularization, act to promote EPC proliferation, differentiation, and mobilization from BM (via the peripheral circulation) to neovascular foci.

ischemia. This appears to result from paracrine upregulation of the principal VEGFR-2 (KDR) receptor in response to factors released from hypoxic skeletal myocytes (16). Receptor upregulation on ECs within the region of lower limb or myocardial ischemia thus enables these cells to act as magnets for any VEGF secreted into the ischemic milieu. Only when VEGF expression is locally protracted at high levels has it been possible to violate this principle (17).

Impact of clinical phenotype on neovascularization. Preliminary clinical findings in patients with critical limb ischemia indicated that the response to phVEGF gene transfer was most robust and expeditious in young patients with premature atherosclerosis involving the lower extremities, so-called Buerger's disease (18). This clinical observation was supported by experiments performed in live animal models, specifically young (4-5 years) versus old (6-8 months) rabbits and young ( 8 weeks) versus old (2 years) mice. In both cases, native neovascularization of the ischemic hindlimb was markedly retarded in old versus young animals. Retardation of neovascularization in old animals appeared in part to result from reduced expression of VEGF in tissue sections harvested from the ischemic limb (19). Similarly retarded neovascularization and reduced VEGF expression was observed in nonobese diabetic (NOD) (20) and hypercholesterolemic (apo E-/-) (21) mice. Cell-specific immunostaining localized VEGF protein expression to skeletal myocytes and infiltrating $\mathrm{T}$ cells in the ischemic limbs of C57 mice; in contrast, VEGF-expressing T-cell infiltrates were found to be severely reduced in ischemic limbs of mice in which angiogenesis was impaired. Transendothelial migration of human $\mathrm{T}$ cells has been shown previously to be compromised in elderly versus young subjects, although the basis for this defect in transmigration remains enigmatic (22). The critical contribution of $\mathrm{T}$ cells to VEGF expression and collateral vessel growth has been reinforced by the finding of accelerated limb necrosis in athymic nude mice with operatively induced hindlimb ischemia (21).

Reduction in endogenous VEGF expression, however, was not the only factor contributing to impaired neovascularization in these animals; older, diabetic, and hypercholesterolemic animals - like patients - also exhibit age-related endothelial dysfunction, manifest as reduced vasodilation and decreased production of nitric oxide in response to endothelium-dependent agonists (19). Endothelial dysfunction did not preclude a favorable response to cytokine replacement therapy: indeed the absolute magnitude by which blood pressure ratio, angiographic score, and capillary density were increased in response to supplemental administration of recombinant VEGF protein was similar for young and old animals. In older animals, however, these indices failed to reach the ultimate levels recorded in younger animals, apparently reflecting the inherent limitations imposed by a less-responsive EC substrate.

This clinical experience and these animal studies have 2 implications. First, the findings suggest that the fundamental mechanism by which therapeutic neovascularization augments collateral development is to provide cytokine supplements to individuals who, because of advanced age, diabetes, hypercholesterolemia, and/or other as yet undefined circumstances, are unable to appropriately upregulate cytokine expression in response to tissue ischemia. In this regard, ligand supplementation may be analogous to erythropoietin administration in patients with refractory anemia.

Second, cytokine administration clearly represents only one aspect of the therapeutic intervention. Regardless of how much ligand is administered, the resident population of ECs competent to respond to an available level of angiogenic growth factors may also constitute a potentially limiting factor in strategies designed to promote neovascularization of ischemic tissues. A reasonable goal may therefore consist of developing a complementary strategy that would provide substrate together with ligand, a "supply side" version of therapeutic neovascularization.

Postnatal vasculogenesis. The option of performing fullscale EC transplantation to optimize this therapeutic strategy is daunting, if even feasible. Accordingly, we investigated an alternative strategy designed to exploit the conceptual notion that ECs and hematopoietic stem cells (HSCs) were ultimately derived from a common precursor, the putative hemangioblast. HSCs had been shown previously to be present in circulating blood, in quantities sufficient to permit their harvesting and readministration for autologous - in lieu of bone marrow - transplantation. We therefore inferred that relat- 
Cell therapy for ischemia

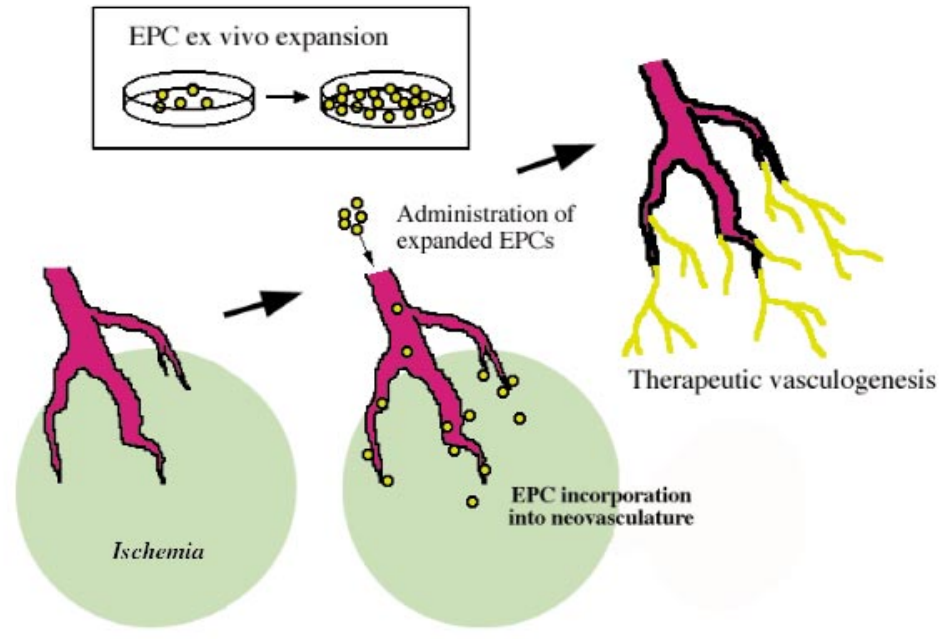

EPC gene therapy for ischemia
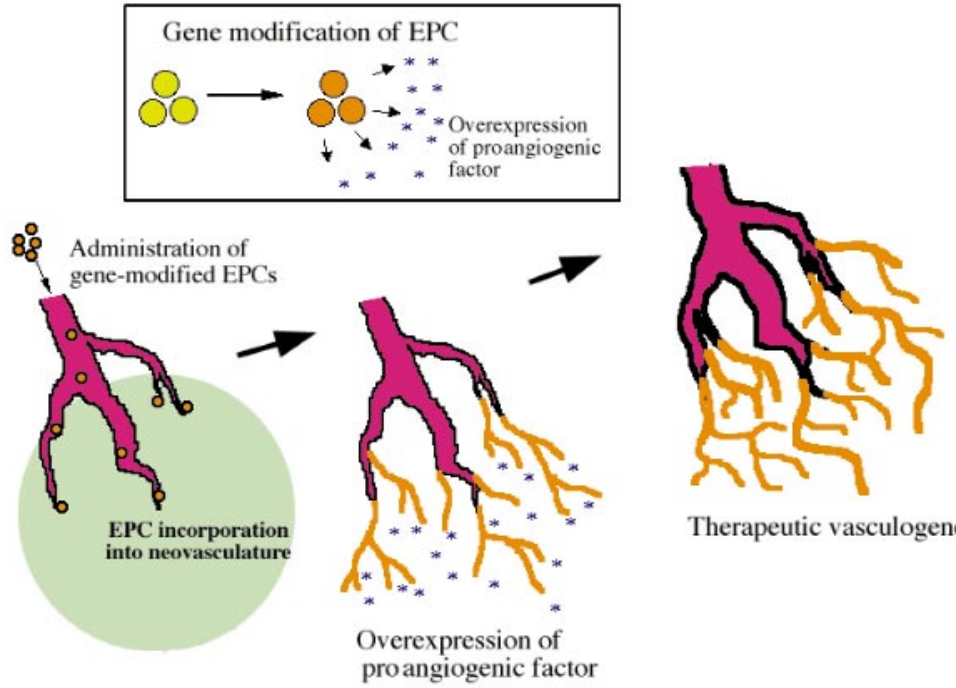

Therapeutic vasculogenesis

ed descendants, endothelial progenitor cells (EPCs), might be present along with HSCs in the peripheral circulation. Flk-1 and a second antigen, CD34, shared by angioblasts and HSCs, were used to isolate putative angioblasts from the leukocyte fraction of peripheral blood (23). In vitro, these cells differentiated into ECs. In animal models of ischemia, heterologous, homologous, and autologous EPCs were shown to incorporate into sites of active neovascularization.

More recently, we have utilized a bone marrow (BM) transplant (T) model to demonstrate incorporation of BM-derived EPCs into foci of neovascularization (Figure 1). Wild-type mice were sublethally irradiated with $9.0 \mathrm{~Gy}$ and transplanted with BM harvested from transgenic mice of the same genetic background in which constitutive LacZ expression is regulated by an EC-specific promoter, Flk-1 or Tie-2. Flk-1 (VEGFR-2) has been shown to be essential for EPC (angioblast) differentiation and blood vessel development during embryogenesis and
Figure 3

Therapeutic vasculogenesis. (top) Circulating EPCs may be harvested in analogous fashion to methods currently established for harvesting HSCs for autologous marrow transplantation. EPCs, purified and expanded ex vivo, may then be readministered, with or without angiogenic growth factors, to optimize therapeutic neovascularization. (middle) Harvested EPCs may be transfected ex vivo with genes encoding for proangiogenic factors. When incorporated into nascent vasculature, administered EPCs express growthpromoting factors directly at the site of, and thereby potentially augment, neovascularization. (bottom) If EPCs are transduced ex vivo with transgene encoding antitumorigenic factors, administered EPCs home to vascular infrastructure of developing neoplasm where they act as a "Trojan Horse" to express antiangiogenic factors that sabotage tumor growth and metastasis.

postnatal neovascularization. The Tie2 receptor has been shown to be expressed in endothelial lineage cells participating in angiogenesis, and in this regard is essential for blood vessel development and maturation. Consequently, $\beta$-galactosidase is constitutively overexpressed in the BM of the transplant recipient Flk-1 or Tie-2/LacZ mice but not in any other somatic cells. Application of a solution of X-gal to the BM renders it blue (Figure 1), and any blue cells that are detected at remote tissue sites can thus be inferred to have been derived from BM and delivered to those sites via the peripheral circulation. After a period of 4 weeks after transplant, by which time the $\mathrm{BM}$ of the recipient mice is reconstituted, a variety of surgical experiments may be performed (Figure 1), all of which are intended to provoke neovascularization. For example, preliminary experiments performed in a mouse model of corneal injury disclosed BM-derived cells incorporated into neovascular foci at the corneal limbus (Figure 1). A similar approach may be used to investigate the contribution of circulating, BM-derived EPCs to neovascularization of ischemic hindlimbs, injured corneas, and tumor vasculature.

Previous reports have established that wound trauma causes mobilization of hematopoietic cells, including pluripotent stem or progenitor cells in spleen, $\mathrm{BM}$, and peripheral blood. Consistent with EPC/HSC common ancestry, recent data from our laboratory has shown that mobilization of BM-derived EPCs constitutes a natural response to tissue ischemia (24). In these experiments, we used the murine BMT model to establish direct evidence of enhanced BM-derived EPC incorporation into foci of corneal neovascularization following the development of hindlimb ischemia. Light-microscopic examination of corneas excised 6 days after micropocket injury and concurrent surgery to establish hindlimb 
ischemia demonstrated a statistically significant increase in cells expressing $\beta$-galactosidase in the corneas of mice with, versus those without, an ischemic limb (24). This finding indicates that circulating EPCs are mobilized endogenously in response to tissue ischemia, after which they may be incorporated into neovascular foci to promote tissue repair (Figure 2).

Therapentic vasculogenesis. Having demonstrated the potential for endogenous mobilization of BM-derived EPCs, we considered that iatrogenic expansion and mobilization of this putative EC precursor population might represent an effective means to augment the resident population of ECs competent to respond to administered angiogenic cytokines. Such a program might thereby address the issue of endothelial dysfunction or depletion that may compromise strategies of therapeutic neovascularization in older, diabetic, and hypercholesterolemic animals and patients. GM-CSF, which stimulates hematopoietic progenitor cells and myeloid lineage cells, as well as nonhematopoietic cells such as BM stromal cells and ECs, was employed to test this notion (24). To effect GM-CSF-induced EPC mobilization while avoiding a direct effect on ECs, recombinant human GM-CSF was administered daily for 7 days prior to surgery to create hindlimb ischemia. GM-CSF pretreatment produced a statistically significant increase in the circulating population of EPCs and enhanced EPC differentiation versus controls. Following surgical development of limb ischemia capillary density analysis documented extensive neovascularization induced by GM-CSF pretreatment, and measurements of ischemic/normal hindlimb blood pressure ratio disclosed evidence of corresponding increase in hindlimb blood flow. These results thus indicate that GM-CSF exerts a potent stimulatory effect on EPC kinetics and that such cytokine-induced EPC mobilization can enhance neovascularization of severely ischemic tissues as well as de novo vascularization of previously avascular sites.

Differential expression of phenotypic markers that permit isolation of EPCs from not only HSCs, but ECs as well, will facilitate strategies of therapeutic vasculogenesis. Antibodies to human CD34 identify a heterogeneous population of cells that includes both HSCs and EPCs. In our early experiments, putative EPCs were first permeabilized with $1 \%$ paraformaldehyde and $1 \%$ Triton X-100, after which EPC selection was achieved using antibodies that recognized the intracellular domain of VEGFR-2 (Flk1). More recently, we have used a monoclonal antibody directed against the extracellular domain of VEGFR-2 (Sigma Chemical Co., St. Louis, Missouri, USA). While VEGFR-2 is generally considered to distinguish EPCs from HSCs, there exists no epitope whose expression is restricted exclusively to EPCs versus fully differentiated ECs. There are at least 3 lines of evidence, however, that suggest that EPCs constitute the preponderance of such circulating, BM-derived endothelial lineage cells. First, previous work has shown that freshly isolated CD $34^{+}$cells display a paucity of EC-specific markers, in contrast to plated cells cultured for 7 days (23). Second, recent work from our laboratory has shown that in contrast to EPCs, heterologously transplanted differentiated ECs rarely incorporate into foci of neovascularization. Third, previ- ous work suggests that the number of differentiated ECs circulating in peripheral blood, identified using P1H12 antibody (25), ranges between 2 and 3 per milliliter, whereas the population of circulating EPCs in normal individuals based on work from our own laboratory is in the range of 0.5 to $1 \times 10^{3}$ per milliliter of blood.

These experimental findings call into question certain fundamental concepts regarding blood vessel growth and development in adult organisms. Postnatal neovascularization has been previously considered synonymous with proliferation and migration of preexisting, fully differentiated ECs resident within parent vessels, i.e., angiogenesis (1). The finding that circulating EPCs may home to sites of neovascularization and differentiate into ECs in situ is consistent with vasculogenesis (26), a critical paradigm for establishment of the primordial vascular network in the embryo. While the proportional contributions of angiogenesis and vasculogenesis to postnatal neovascularization remain to be clarified, our findings, together with recent reports from other investigators $(27,28)$, suggest that growth and development of new blood vessels in the adult is not restricted to angiogenesis but encompasses both embryonic mechanisms. As a corollary, augmented or retarded neovascularization, whether endogenous or iatrogenic, likely includes enhancement or impairment of vasculogenesis.

Moreover, the observation that circulating EPCs home to foci of neovascularization suggests potential utility as autologous vectors for gene therapy (Figure 3). For treatment of regional ischemia, neovascularization could be amplified by transfection of EPCs to achieve highly localized constitutive expression of angiogenic cytokines and/or provisional matrix proteins. For antineoplastic therapies, EPCs could be transfected with, or coupled to, antitumorigenic drugs or angiogenesis inhibitors.

Future studies will clarify the mechanisms and circumstances that may be responsible for modulating the contribution of vasculogenesis to postnatal neovascularization. Specifically in this regard, it is intriguing to consider the possibility that certain angiogenic growth factors that are acknowledged to promote both angiogenesis and vasculogenesis in the embryo, but have been assumed to promote neovascularization exclusively by angiogenesis in the adult, may in fact promote migration, proliferation, and mobilization of EPCs from BM. Finally, the possibility that modulation of vasculogenesis can be used therapeutically to augment, as well as inhibit, neovascularization deserves further investigation.

\footnotetext{
1. Folkman, J. 1971. Tumor angiogenesis: therapeutic implications. N. Engl. J. Med. 285:1182-1186.

2. Takeshita, S., et al. 1994. Therapeutic angiogenesis: a single intra-arterial bolus of vascular endothelial growth factor augments revascularization in a rabbit ischemic hindlimb model. J. Clin. Invest. 93:662-670.

3. Ware, J.A., and Simons, M. 1997. Angiogenesis in ischemic heart disease. Nat. Med. 3:158-164.

4. Arras, M., et al. 1998. Monocyte activation in angiogenesis and collateral growth in the rabbit hindlimb. J. Clin. Invest. 101:40-50.

5. Mack, C.A., et al. 1998. Biologic bypass with the use of adenovirus-mediated gene transfer of the complementary deoxyribonucleic acid for vascular endothelial growth factor 121 improves myocardial perfusion and function in the ischemic porcine heart. J. Thorac. Cardiovasc. Surg. 115:168-176.

6. Losordo, D.W., et al. 1994. Use of the rabbit ear artery to serially assess foreign protein secretion after site specific arterial gene transfer in vivo: evidence that anatomic identification of successful gene transfer may underestimate the potential magnitude of transgene expression. Circulation. 89:785-792.
} 
7. Murohara, T., et al. 1998. Nitric oxide synthase modulates angiogenesis in response to tissue ischemia. J. Clin. Invest. 101:2567-2578.

8. Wang, H., and Keiser, J.A. 1998. Vascular endothelial growth factor upregulates the expression of matrix metalloproteinases in vascular smooth muscle cells: role of flt-1. Circ. Res. 83:832-840.

9. Isner, J.M., et al. 1996. Clinical evidence of angiogenesis following arterial gene transfer of phVEGF ${ }_{165}$. Lancet. 348:370-374.

10. Baumgartner, I., et al. 1998. Constitutive expression of phVEGF $_{165}$ following intramuscular gene transfer promotes collateral vessel development in patients with critical limb ischemia. Circulation. 97:1114-1123.

11. Losordo, D.W., et al. 1998. Gene therapy for myocardial angiogenesis: initial clinical results with direct myocardial injection of phVEGF $_{165}$ as sole therapy for myocardial ischemia. Circulation. 98:2800-2804.

12. Schumacher, B., Pecher, P., von Specht, B.U., and Stegmann, H. 1998 Induction of neoangiogenesis in ischemic myocardium by human growth factors: first clinical results of a new treatment of coronary heart disease. Circulation. 97:645-650.

13. Namiki, A., et al. 1995. Hypoxia induces vascular endothelial growth factor in cultured human endothelial cells. J. Biol. Chem. 270:31189-31195.

14. Shen, B-Q., et al. 1998. Homologous upregulation of KDR/Flk-1 receptor expression by vascular endothelial growth factor in vitro. J. Biol. Chem. 273:29979-29985.

15. Fujio, Y., and Walsh, K. 1999. Akt mediates cytoprotection of endothelial cells by vascular endothelial growth factor in an anchorage-dependent manner. J. Biol. Chem. In press.

16. Brogi, E., et al. 1996. Hypoxia-induced paracrine regulation of VEGF receptor expression. J. Clin. Invest. 97:469-476.

17. Springer, M.L., Chen, A.S., Kraft, P.E., Bednarski, M., and Blau, H.M.
1998. VEGF gene delivery to muscle: potential role for vasculogenesis in adults. Mol. Cell. 2:549-558.

18. Isner, J. M., et al. 1998. Treatment of thromboangiitis obliterans (Buerger's disease) by intramuscular gene transfer of vascular endothelial growth factor: preliminary clinical results . J. Vasc. Surg. 28:964-975.

19. Rivard, A., et al. 1999. Age-dependent impairment of angiogenesis. Circulation. 99:111-120.

20. Rivard, A., et al. 1999. Rescue of diabetes related impairment of angiogenesis by intramuscular gene therapy with adeno-VEGF. Am. J. Pathol. 154:355-364.

21. Couffinhal, T., et al. 1999. Impaired collateral vessel development associated with reduced expression of vascular endothelial growth factor in $\mathrm{ApoE}^{-/-}$mice. Circulation. In press.

22. Stohlawetz, P., et al. 1996. The effect of age on the transendothelial migration of human T lymphocytes. Scand. J. Immunol. 44:530-534.

23. Asahara, T., et al. 1997. Isolation of putative progenitor endothelial cells for angiogenesis. Science. 275:965-967.

24. Takahashi, T., et al. 1999. Ischemia- and cytokine-induced mobilization of bone marrow-derived endothelial progenitor cells for neovascularization. Nat. Med. 5:434-438.

25. Solovey, A., et al. 1997. Circulating activated endothelial cells in sickle cell anemia. N. Engl. J. Med. 337:1582-1590.

26. Risau, W., et al. 1988. Vasculogenesis and angiogenesis in embryonic stem cell-derived embryoid bodies. Development. 102:471-478

27. Shi, Q., et al. 1998. Evidence for circulating bone marrow-derived endothelial cells. Blood. 92:362-367.

28. Hatzopoulos, A.K., Folkman, J., Vasile, E., Eiselen, G.K., and Rosenberg, R.D. 1998. Isolation and characterization of endothelial progenitor cells from mouse embryos. Development. 125:1457-1468. 